\title{
ALIMENTACION ARTIFICIAL EN EL LACTANTE SANO
}

\author{
Por el Dr. Jullo A. BAUZA. \\ Diretor de la División Primera Infancia $y$ de la Casa dei Niña \\ Montevideo.
}

Nos parece actualmente lejana la época, en la cual se miraba a la alimentación artificial como algo poco menos que imposible de tealizar con éxito: Sin dejat de reconocer. abora como siempre, a la alimentación del niño por al pecho de la madre, como el desideratum al que debe el médico aproximarse la más posible, es indudable que la apticud de las madres para criar, tiende a reducirse, a juzgar por la impresión que obtenemos de nuestra práctica diaria. Por tanto. el inédico se ve forzado precozmente a recurtir a la alimentación nixta o al alimento artificial.

Entendemos que los adelantos de los últimos 30 años han llegado poco a poco a permitir al médico experto. Ia posibilidad de la crianza de niños sanos con jlimento artificial, con resultados comparables a los que pueden ser obtenidos en la ajimentación a perho.

Convengamos también que estos resultados sólo pueden ser alcanzados en el medio familiar, en el que el niño es cuidado, siempre por la misma persona, día $y$ noche y durante todos los días de la semana, lo que representa un factor psíquico de importancia decisiva para su buen resultado.

La alimentación artificial del lactante sano.

Las bases para la alimentación artificial son de todos conocidas.

$1 .^{9}$ Allimento cuantitativamente suficiente.

2. Alimento cualitativamente apropiado. cuyos cumponentes guarden una relación que no se aparte fundamentalmente de la que se vbserva en la leche humana. 
3. Alimento que contenga en cantidad suficiente las vitaminas y otros elementos indispensables para una normal nutrición.

4. Alimento libre de gérmenes patógenos.

Cumplidas estas reglas fundamentales. las reglas accesorias sun:

1." Mantenimiento de los intervalos necesarios para una buena digestión.

2.9 Aplicación de procedimientos que aumentén la digestibilidad del alimento.

3." Suministro del alimento a la emperatuta de! cuerpo humano.

Con la primera de las reglas fundamanta!es queremos decir que el alimento debe 1lenat las exigencias calóricas del niño, o sea, de 110 a 100 cal. por kilogramo de peso en el primer trimestre, de $100 \mathrm{cal}$. en el segundo trimestro y de $90 \mathrm{cal}$. en el tercero y cuarto.

Con la segunda, o ley de la correlación de los componentes, ley de la armonía de Escudero, queremos significar que, en términos generales debe mantenerse la relación de 1:3:6 entre las proteinas, grasas e hidratos de carbono. pudiendo, no obstante, admitirse una modificación en lo re. ferente a la relación proteína a hidrato de carbono, que en la leche de vaca pura es de 1 a 1,5 . mediante la desamortiguación de la caseína, y el aumento de hidratos de C., procurando hacer esta relación más elevada. llevándola a $1: 3$, mediante el agregado de hidratos de carbono. En este principio, colocamos igualmente el contemplar que se llenen las necesidades de agua del organismo, suministrándose de 150 a $160 \mathrm{grs}$. por $\mathrm{kgr}$. de peso.

En cuanto a la tercera regla fundamental, consideramos de extraordinaria importancia, que el alimento artificial contenga toras las vitaminas necesarias al niño en cantidad óptima.

La vitamina $A$ no constituye un problema, ya que en la forma corriente de alimentación se asegura una cantidad completamente suficiente.

Lo mismo podemos decir de la vitamina BI, que la leche de vaca contiene en proporción algo mayor que la de mujer. En lo referente a $\mathrm{B} 2$ y al complejo vitamínico conexo, no hay en general deficiencia a señalar.

Es sabido que el intestino del niño a pecbo sintetiza la vitamina $\mathrm{Bl}$ por la acción del bifidus. $y$ que las materias 
fecales del niño a pecho pueden curar el beriberi de las palomas en pocas horas. Pero el bifidus desaparece con el cam. bio de acidez del medio intestinal. y con ello la capacidad de sintetizar dicha vitamina. Esta deficiencia puede ser compensada, por la riqueza en $\mathrm{Bl}$ de la leche de vaca bien alimentada. En caso de duda, y en consideración a la inocuidad de la vitamina $\mathrm{Bl}$, no habría inconveniente en que fuese suministrada a clertos lactantes.

En lo referente a la vitamina $C$. consideramos indispensabíes el suministro de dosis no inferiotes de 25 milígrames diarios de ácido ascórbico, o de $50 \mathrm{cc}$. de jugo de naranja para prevenir la hipovitaminosis $C$.

Más importancia tiene el suministro de la vitamina $D$, la cual debe ser administrada a todo niño alimentado artificialmente. si es posible, bajo forma de aceite de hígado de bacalao (3 cucharaditas al día, o sea, 1,000 U. I.). o mejor aún, bajo forma de aceite de hígado de percomorfo enriquecido en vitamina $D$. No debemos dejar de considerar que el aceite de hígado de los peces de esta familia, contiene, además de vitamina $D$, pequeñas cantidades de yodo, dr que tiene necesidad el organismo del niño alimentado artiticialmente. El suministro de vitamina $D$, con la observancia de las reglas higiénicas elementales, de vida a plena luz y buena ventilación, tienen una importancia decisiva en la profilaxis del raquitismo.

La pobreza de hierro en la leche de vaca debe ser tenda en cuenta para prevenir la aparición de una anemia ferripriva. Entendemos y acostumbramos a hacerlo, que todo niño aitmentado artificialmente debe recibir una pequeña dosis de hietro (citrato de hierro amoniacal, $0,20 \mathrm{gr}$. por día).

Finalmente, la cuarta regla fundamentai os que el allmonto esté libre de gérmenes patógenos, ya que el estreptzcoco, el colibacilo, el bacillus disentérico y las salmonellas. al infectar el alimento, van a llevar al intestino del niño géxmenes cupa acción deletérea no tardará en manifestarse.

Las reglas accesorias, son casi tan importantes come las precedentemente citadas. El mantenimiento de los intervalos que requiere la digestión del alimento, es como mínimo de 3 horas. Intervalos de este tiempo entre una comida y otra deben ser estrictamente mantenidos en el primer trimestre, y mismo hasta el $4 .^{\circ}$ mes. At comienzo del $59^{\circ}$ mes y a veces antes, es posible y asi mismo conveniente particularmente en 17 estación calurosa, establecer el régimen de 5 comidas, con 
intervalos de 4 horas, y mismo de 4 cumidas después de los 6 meses en los niños con tendencia a la obesidad.

Procurar un alimento que aumente el grado de digestibilidad de la leche de vaca, y con ello una mejor tolerancia, debe ser la suprema aspitación del médico, ya que el estómago del niño no está constituido como el de los vacunos para digerir la leche de vaca, existiendo con el del nin̈o diferencias anatómicas fundamentales.

Procurar obtener para la digestión gástrica, condiciones de acidez que se aproximen a la óptima, que se presenta en la digestión de la leche de mujer. significa asegurar el buen exito a cue se aspira. En tanto no se trate de niños con hiperclorhidria, o cuyo estado permita suponer que podrăn compensar la difícil digestión de la leche de vaca con mayor aporte de jugo gástrico el médico jsuministrati apenas se presenten signos de disminución de la tolerancia, un alimento parcialmente desamortiguado, por acidificación. que per mita una previa coagulación de la caseína en finos copos facilmente digeribles, y con las demás condiciones que faciliten su más fácil disgregación en el estómago del nińo.

Para nosotros no cabe duda de que la desecación de la leche. al prisrorcionar un alimento de composición invaria. ble de escasa atterabilidad por una parte, y la acidificación por el agregado de ácidos orgánicos por otra, presentan dos medios de insospechadas proyecciones en lo jeferente a la alimentación artificial.

La desecación de la leche, pone al alcance de la madre un producto de composición invariable, de fácil e inmediata preparación, de asepsia segura. A nuestro modo de ver, al niño sano, después de habérsele suministrado alimento natural exclusivo durante el primer mes, se le puede alimentar parcial o totalmente con leche desecada o productos detivaclos, a la condición de que este alimento le sea suministrado cumpliéndose las reglas fundamentales y accesorias que henos enunciado.

Especial atención deberá prestarse al agreg ido de hiaratos de carbono en lo teferente a su cantidad y calidad.

En lo referente a lo primero. los $\mathrm{H}$. de carbono a agregarse deben constituir del 7 al 8 por 1,000 grs. de peso, cantidad a subdividirse en las 5 ó 6 comidas a administrarse. Tal seria el caso para los niños a alimentarse con leche en polvo semi-descremadas (Dryco, alacta, Bebelet semidescremadol que no tienen otro $H$. de carbono que el que naturalmente contiene ta leche. 
Otro sería el caso para aquellos niños, en cuya alimentación entrarían polvos de leche con agregados de azúcares de distinta indole (lactosa, sacarosa, dextromaltosa), en cuyo caso, sólo será necesaria la simple dilución.

En cualquieta de ambas situaciones, el médico puede hacer la dilución con un mucílago, en lugar ce agua simple. o unismo de una suspención a concentración mayor. por ej., suspensión acuosa de maizena al $2 \%$ procedimientos que tienen su valor al objeto de facilitar la digestión del producto, y una mejor fijación de agua. En los casos de tratars: de leches desecadas con azúcar (Lactógeno, Nestógeno, Prolaka), aconsejamos concentraciones de $14 \%$.

Para el cátaula de la ración, pueden sezuirsa d)s prosedimientos:

1. Reconstituir, mediante la dilución de la leche en polvo $(11 \%$; si se trata de leche semidescremada: $12.5 \%$. si se trata de las llamadas leches secas integrales), el producto que se desea suministrar. Se agtegará luego el agua que se desee, a los efectos de la mayor o menor dilución que se precura, y finalmente el $\mathrm{H}$. de carbono en id calidad $\mathrm{y}$ camtiada que se quiera.

Si se trutase de un niñn a ser alimentado con leche en polvo, ya enriquecida con $H$. de carbono, se iniciará con una dilución al 12 por 100 para elevar sa concentración al $14 \%$ después del segundo mes.

Si se quiere aplicar el método de las calorias, al que damos la preferencia, se comenzatá por fijar el cuantum calórico que se quiere suministrar al día. Se deducitá (caso de leche sin agregado de $\mathrm{H}$. de carbono) el valor energético correspondiente al azúcar a ser agregado, a sazón de 7 a 8 grs. por kgr. de peso; la diferencia será en calorias lo que deberá ser aportado por la lechée seca. Calculando a tazón de 4,5 cal, por gramo de leche seca semidescremado, y de 5 cal. para un gramo de integral, es fácil calcular la cantidad en gramos necesaria para constituir la ración diaria. Las cifras obtenidas, tanto para azúcar como para leche seca, serán divididas por 6 , si se han de suministrar 6 raciones: por 5 , si se prefiere el régimen de 4 horas con 5 comídas. E1 diluyente, agua, o mucílago, será calculado a razón de. 150 a 160 grs. por kilogramo de peso, diario.

Veamos un caso: Niño de 1 mes, de 4 kilogrames de peso, a ser alimentado con Dryco. Le corresponden a un coeficiente de 100 cal, por kilogramo, 400 cal. diarias que repartiremos así: 
Dextromaltosa, u otro azúcar, 7 grs. por kilogramo, igual 28 grs. diarios, o sea, redondeando, 5 grs. por vez, a régimen de 6 raciones. Valor calórico aproximado, 120 cal.

Restan 280 calorías a ser suministradas por la leche seca semidescremada. Esta cifra dividida por 4.5 da en gramos la ración diaria de leche seca; para este caso, que seria de 60 grs., cantidad que dividida por 6 , número de raciones diarias nos da 10 grs. En cuanto al agua, a 160 por kilogramo de peso, nos daría 640 grs., o sea, para cada biberón.

\section{Agua: 110 grs. \\ Azúcar: 5 grs. \\ Leche seca semidescremada: 10 grs.}

Cono cada medida, de las que traen, por lo general. los tarres de leche seca, son. de 5 gramos, habría que disolver 2 medidas del producto láicteo desecado y 1 cuchara ${ }^{\prime}$ ta de azúcar en 110 grs. de agua hervida o de un mucílago de avena o de otra indole.

Determinada la ración óptima se verá cómo responde e) niño a ella, de acuerdo a caracteristicas individuales de su metabolismo.

Al tercer mes se podrá usar la leche desecada integral, que en la ditución de $10 \%$ provee un producto con 2,2 a $2,4 \%$ de manteca y a las que se agregará de 5 a 7 \% de azúcar o de dextromaltosa.

En cualquiera de ambos casos, puede set úti1. para no aumencar exageradamente la concentración y con ello la proporción de caseína, de agregar una mezcla mantecofarinázea al 3 por 100 de harina y 3 por 100 de manteca, o bien diluir la leche seca semideseremada en una papilla manteco. farinácea de la fórmula $3-3-5$ por 100 , o seà, de $3 \mathrm{grs}$. de marteca, 3 grs. de hłrina y 5 grs, de azúcar, con ura proporción de leche en polvo de 8 a $10 \%$.

Como se ve, el suministro de la leche en polvo permite un sin número de modificaciones, así como la del aumento de la concentración, suministrándose entre horas el déficit de agua que fuese la consecuencia de la aplicación de este procedimiento (casos de píloroespasmo. por ej.).

Las leches acidificadas: Prescindiendo del empleo. más que centenario del babeurre, o alimento holandés, aplicado para el tratamiento de los trastornos digestivos del lactant? hace. 150 años. hecho conocer en Alemania en 1902 por Texeira de Matto, puede decirse que recién hace 25 años qute 
su empleo se ha generalizado en la práctica de la alimentación artificial.

Marriot y Davidon, a raíz de sus estudios sobre concentración hidrogeniónica del contenido gástrico, durante la digestión de la leche de mujer y de la leche de vaca, han demostrado la más fácil digestibilidad de las lechrs acidificadas con ácido láctico, al permitir un $\mathrm{pH}$ gástrico más aproximado al de la digestión de la leche de mujer. favorable a la acción de la pepsina, en los niños lactantes alimentados con leche acidificada, así como una evacuación más rápida del estómago como consecuencia de lo mismo.

A la mayor aciđez del contenido gástrico habia que agregar la acción antiséptica que es su resultante. Para Faber. cl ácido actuaría favorablemente sobre la lipasa gástrica.

No es nuestro propósito detallar agui, los distintos procedimientos que se emplean para la acidificarión de la leche. limitándonos a señalar los buenos resultados que se obienen medrante el agregado de ácido láctico, 5 c.c. por 1,000 de leche pura, o sea, un gramo por cada 200 grs. de leche $(1$ cc. de ácido láctico equivale a 28 ó 30 gotas de los cuenta gotas corrientes. o a 2 cucharitas de $5 \mathrm{grs}$. $c / \mathrm{u}$. de una solución acuosa al $10 \%$ de este, producto).

El agregado de $2 \%$ de maicena y de $6 \%$ de dextromatosa, facilita la tolerancia del alimento y aumenta poderosamente su valor energética, una de las mayores virtudes de este yénero de alimento, llevando su rendiniento a 900 cal. por litro, o sea, de 90 cal, por cada 100 grs. En sir aplicación se usará de 100 a 120 grs, por kilogramo de peso. si se trata de niños sanos de peso normal. Para los distróficos se calcularán 150 grs. por kilogramo de peso red, o bien, la dosis anteriormente citada por kilogramo de pese debido, - sea, el correspondiente a su edad. Se mantentrá la ración de agua dentro de las necesidades fisiológicas y de acuerdo a la estación del año. Las leches acidificadas con jugo de limón a la dosis de $28 \mathrm{cc}$. por 1.000 grs. o de 40 cc. como lo hemos aconsejado. en atención a la menor acidez de los limones, en nuestro país.

L a acidificación de la leche destinada a la alımentación del lactante. puede ser igualmente aplicada a la leche an polvo, con la ventaja de aplicarse a un producto parcialmente desamortiguado por el proceso de la desecación, especialmente si la desecación se ha hecho mediante el método "roller" que modifica profundamente la caseína como consecuencia de la temperatura en los cilindros. Además, se 
tienen en tal caso todas las ventajas ya señaladas, del empleo de la leche desecada.

La alimentación con las leches acidificadas artiticial. mente, no tiene por qué, a nuestro juicio, ser aplicada como rutina de alimentación, sino reservada pata aquellos casos de niños, en los cuales puede suponerse un cierto grado de insuficiencia digectiva, o que on la estación calurosa o en cualquier época del año presentan signos de pre-dispepsia. que petmita preveer la posibilidad de la aparición de un trastorno nutritivo.

El médico que dirige la alimentación artificial del niño deberá considerar que la leche acidificada con ácido láctico es más desamortiguada o más antidispéptica que aquélla a la que se agrega jugo de limón.

Esta última es rica en vitamina $C$, en tanto que a la anterior se hace imprescindible su agregado en la cantidad suficiente.

Taxibién se deberá tener presente, que si la digestión gárical es tacilitada mediante la acidifiración, eft el intestinis no se observará igual modificación. Er. efecte. !as evacuacicnes dz estos niños, son alcalinas como en aquellos que reciben la leche común a la misma concentración, no siendo maycrmente influenciado el pH intestinal, que en el intestino grueso, dependerá de la relación existente entre sustancias putrefactivas (proteinas) y sustancias fermentescibles (hidratos de carbono, particularmente lactosa). Sólo el agregado de una elevada cantidad de lactosa, no inferior a 12 por 100 , permitiria, como to comprobó Gerstley, con Howel y Nagel contrarrestar el afecto alcalinizante y cambiar la flora Gram negativo a una flora Gram positivo, sinilat a la que se observa en el niño de pecho.

Ahora bien, el agregado de una cantidad de lactosa tan elcvada, puede no estar desprovisto de inconvenienre, además de ser inseguro en el resultado que se procura obtener.

Por otra parte, queda en pie la pregunta de si el entpleo largo tiempo prolongado de la leche acidificada al ácido láctico, no podría originar un drenaje de álcali de los tejidos, capaz de favorecer la aparición del raquitismo, como ha sido señalado en el "daño por la leche de vaca". En todo caso, no bay por qué prolongar exageradamente el uso de la leche acidificada, ya que creciendo ei niño en edad $v$ con ello aumentando su tolerancia a la leche, no habria 
mayor inconveniente en pasar a un régimen de alimentc con leche no acidificada.

Para la acidificación de la leche con ácido láctico, puede partirse de la leche fresca hervida, o bien de la leche en polvo, pero resultados particularniente favorables se señalan si se emplea la leche evaporada, actualmente tan en boga en Estados Unidos y en Cuba.

Para la alimentación del lactante con leche evaporada se requiere su dilución al tercio, agregándole la proporción de hidratos de carbono necesaria, ya que el productos industrial no los contiene. En ciettos casos puede usarse la dilución con agua en partes iguales, con lo cual se obtiene un producto de la composición de la leche de vaca pura. E1 empleo de una mezcla con dextrina y maltosa o de jarabe de maíz, como el producto llamado Caro, resuita más ventajoso que el uso del azúcar común. La cantidad de ácido láctico a agregarse es de 5 gramos por mil. Entre nosutros, este género de alimentación no ha podido aún ser utilizado, ya que las usinas que preparan productos lácteos, no han considerado hasta el presente su preparación como un éxito industrial.

Aspecto científico del problema de la alimentación artificial en los organismos de protección cerrada.

Ei resultado de la alimentación artificial en, los organismos de protección cerrada (hospitales de lactantes, creches, casas-cunas), no es en términos generales tan satisfactorio como pareceria lógico esperar.

Los cambios frecuentes del personal, exigidos por el cumplimiento de las leyes que rigen el trabajo, el descanso obligatorio después de 6 días de labor. el descanso anual reglamentario, las licencias por razones de salud, y otras dificultades de toda índole, traban la eficiencia que se procura conseguir mediante un sistema de organización al oarecer irreprochable.

Como consecuencia de ello, se crean serios inconvenientes a la labor científica, inconvenientes que pueden llegar hasta hacer fracasar los propósitos de investigación del médico que se propone llevar a la práctica nuevos procedimtentos que exigen un cambio en la rutina de la alimentación artificial.

No obstante, el problema de la alimentación artificial, sólo puede ser estudiado científicamente en las instituciones de protección cerrada, ya que en ellas cada caso puede ser 
sometido a un contralor imposible de realizar. cuando el niño es atendido en el ambiente familiar.

Se comprende que para llegar a un resultado, es indispensable disponer de un laboratorio bien montado $y$ de un personal científico que colabore eficientemente dentro del plan que se ha trazado.

Así puede explicarse, que el profesor G. Besau pueda haker conseguido, luego de más de 300 ensayos. preparar un alimento cuya principal característica es la de permitir en el intestino del lactante el desarrollo de una flora a bifidus, idéntica a la que se observa en el niño alimentado a pecho.

Tanto en la clínica de lactantes de la Charité de Berlín. como en la Institución Victoria Augusta, los resultados obtenidos con este nuevo alimento fueron tan halagadores. como tuve oportunidad de verlo en la visita realizada en 1938, que no dudé a mi regreso a Montevideo, en ensayario en la Casa del Niño. El resultado obtenido, aun cuando no tan brillante como el que habiamos observadó, vino a demostrat la exactitud del concepto de Bessau, ya que tanto el $\mathrm{pH}$ intestinal, como la flora observada en las heces, de. mostraton que fisiológicamente este alimento daba lugar en el intestino del niño, a un proceso similar al que normalmente se observa en la digestión de la leche de mujer.

Dificultades de distinto orden nos impidieron llevat adelante la aplicación de este nuevo preparado en mayor escala, habiendo informado sobre su resultado en un estudio presentado a la socieóad de Pediatría de Montevideo y puElicado en los "Arch. de Pediatría del Uruguay" (Tomo $X$. N.: 6. Junio 1, 39).

El resultado obtenido confirmó la afirmación de Bessau, de que con este alimento era posible conseguir una desviación hacia la acidez en el contenido del intestino grueso con un $\mathrm{pH}$ comparable al que se observa en el niño a pecho y con la flora de bifidus que le corresponde.

La preparación de este producto dietético es bastante compleja, y por el momento reservada a un terreno puramente científico.

Como lo dice con razón Bessau, no podrá nunca alcanzarse la preparación de un producto artificial biológicamente idéntico a la leche de mujer, debiéndonos conformar con un sustituto lo más semejante que sc pueda lograr.

Desde el punto de vista práctico, es indudable que, alimentos de esta índole son más artificiales aún que las mez- 
clas lácteas comunes, con las cuales se consigue casi siempre un resultado satisfactorio en los casos en que la alimentación natural no sea posible de realizar.

A los factores de orden interno ya señalados precedenIemente, que conspiran en los internados contra el buen resultado que se espera conseguir, se agrega todavia el factor psicológicu, derivado del ambiente propio a toda institución de protección cerrada, del gran número de nin̄os en la misma sala, de la posición horizontal en que el niño es mantenido en la cama casi todo el día, por insuficiencia de personal. de la ventilación deficiente del local, particularmente en invierno, y finalmente de la forma diferente en que el niño es tratado por las distintas personas que han de ofrecerle el alimento, y que se cambian 3 veces al día, sin contar con al cambio semanal de rigor.

La experiencia recogida en 16 años en la Cuna de la Lasa del Niño de Montevideo, institución que alberga diariamente un promedio de 60 niños menores de 1 año, más de la mitad de los cuales están sometidos a alimentación mixta o artificial, nos autoriza a destacar la superioridad de la leche acidificada sobre la leche de vaca simplemente diluida, y de la Jeche en polvo sobre la leche fresca, para los institutos similares.

El babeurte en polvo diluído al $10 \%$, con un contenido de $1,5 \%$ de materia grasa, y con el agregado de $5 \%$ ce azúcar o de partes iguales de sacarosa y de destromaltosa, con el aditamento de harina de trígo o de maizena, constituye el método corriente de iniciación de alimentación artificial en el lactante de menos ce 6 meses. Cuando se considera rucesario una mayor proporción de materia grasa, se ag:ega al babeurte de 10 a 15 por 100 de una mezcla mantecofarinácea. es decir una sopa de Czerny en babeurre. También señalamos con agrado los buenos resultados obtenidos con la leche ácida hipergrasosa de Gaing, lo que está de acuerdo con el concepto ya conocido de que la acidez del alimento aumenta la tolerancia por las grasas.

Otra forma de alimentación con leche ácida en polvo se aplica mediante el empleo de la leche seca semidescremada o integral, diluída al $12 \%$, con el agregado de $6 \%$ de azúcar o de dextromaltosa y 4 grs. por litro de ácido láctico. Este producto equivale al preparado por la Casa Nestlé con el nombre de Pelargón semidescremado o integral y su empleo nos ba proporcionado resultados excelentes. 
Como se comprenderá, en todos los casos de aliment?ción artificial, no podrá omitirse el agregado de vitamina $C$ y de vitamina $D$, aun cuando la generalización de las leches secas irradiadas pudiera hacer innecesario una mayor cantidad de esta última vitamina.

Si bien es cierto que las propiedades antidispépticas de la leche al ácido láctico son superiores a la leche al jugo de limón $(40$ grs. por litro, pH 5,3$)$, esta última constituye un medio de acidificación de la leche de vaca, de corriente aplicación. particularmente después del $60^{\circ}$ mes, y de cuyos resultados nos declaramos en general muy satisfechos. La fina coagulación de la caseína, su acidez menos pronunciada que en el babeurte, su sabor agradable, aumentan su tolerancia. Es habitual el agregado de $2 \%$ de maizena y de 5 por 100 de azúcar. Su fácil preparación, sea que se parta de la leche seca, como que se emplee la leche fresca, la hacen particularmente indicada.

- Pasado el primer semestre y fuera de la estación calurosa, no vemos inconveniente en el uso de las papillas a base de leche de vaca en estado fresco.

De lo que no debemos apartarnos en ningún caso es. del estudio de la constitución del lactante, cuyo alimento debe instituirse. El resultado bueno o malo a obtenerse, depende particularmente de la constitución del niño, y desde este punto de vista hay que señalar que no siempre es el niño normaí aquel con el cual deberá enftentarse el médico y que su fracasos serian mayotes que los éxitos, si aplicase a todos ei mismo standard de alimentación. 